\title{
NEW DISTORTION THEOREMS FOR FUNCTIONS OF POSITIVE REAL PART AND APPLICATIONS TO THE PARTIAL SUMS OF UNIVALENT CONVEX FUNCTIONS
}

\author{
S. D. BERNARDI
}

ABSTRACT. New distortion theorems are obtained for the class of functions $p(z)=1+c_{n} z^{n}+\cdots(n \geq 1)$ which are analytic and $\operatorname{Re} p(z)>a$ $(0 \leq a<1)$ in the unit disk $|z|<1$. These are used to obtain new results regarding the partial sums of univalent convex functions.

1. Introduction. Let $P_{a, n}$ represent the class of functions $p(z)=1+$ $c_{n} z^{n}+\cdots(n \geq 1)$ which are analytic and $\operatorname{Re} p(z)>\alpha(0 \leq \alpha<1)$ for $z \epsilon$ $E\{z:|z|<1\}$. The class $(K)$ consists of those normalized functions $f(z)=$ $z+a_{2} z^{2}+\cdots$ which are analytic and univalent in $E$ and have convex image domains $f(E), S_{n}(z)=z+a_{2} z^{2}+\cdots+a_{n} z^{n}$ are the partial sums of $f(z)$. Theorems 1, 2 represent extensions of known distortion theorems of functions of positive real part; these are used to derive Theorems 3, 4 which represent new results concerning the partial sums of univalent convex functions.

2. Theorems and their proofs.

Theorem 1. Let $p(z) \in P_{a, n^{*}}$ Then for $|z|=r<1$, and $n=1,2,3, \cdots$,

$$
\left|z p^{\prime}(z)\right| \leq 2 n r^{n} \operatorname{Re}[p(z)-\alpha] /\left(1-r^{2 n}\right) .
$$

For each $n$ and each $a$, equality is attained at $z=r$ for the function $p(z)=$ $\alpha+\left[(1-\alpha)\left(1-z^{n}\right) /\left(1+z^{n}\right)\right]=1-2(1-\alpha) z^{n}+\cdots$.

Proof. The special case of (1) when $p(z) \in P_{0,1}$ is well known. (For a simple proof see remark in $[1, \mathrm{p} .316]$.) For $p(z) \in P_{0, n}$ the weaker inequality $\left|z p^{\prime}(z) / p(z)\right| \leq\left[2 n r^{n} /\left(1-r^{2 n}\right)\right]$ was proven by T. H. MacGregor [4]. To prove (1) we employ a technique similar to that used in [4].

Assume $\alpha=0$, for then the transformation $p(z) \rightarrow(p(z)-\alpha) /(1-\alpha)$ will give the general result. If $p(z) \in P_{0, n}$ then $k(z)=(1-p(z)) /(1+p(z))=$ $d_{n} z^{n}+\cdots$ is analytic for $|z|<1$ and $|k(z)|<1$. Hence $k(z)=z^{n} \phi(z)$,

Received by the editors July 2, 1973.

AMS (MOS) subject classifications (1970). Primary 30 A 32.

Key words and phrases. Univalent functions, convex functions, radius of starlikeness, functions with positive real part. 
where $\phi(z)$ is analytic for $|z|<1$ and $|\phi(z)| \leq 1$. For such functions we have $[5$, p. 168]

$$
\left|\phi^{\prime}(z)\right| \leq\left(1-|\phi(z)|^{2}\right) /\left(1-|z|^{2}\right) \quad(|z|<1) .
$$

From $z^{n} \phi(z)=(1-p(z)) /(1+p(z))$ we obtain
(a) $|\phi|^{2}=\left(1 / r^{2 n}\right)|(1-p) /(1+p)|^{2}$,
(b) $\left|\phi^{\prime}\right|=\left(1 / r^{n+1}\right)\left|\left(2 z p^{\prime}+n\left(1-p^{2}\right)\right) /(1+p)^{2}\right|$,

where $r=|z|, p \equiv p(z)$ and $\phi \equiv \phi(z)$. Substituting (a) and (b) into (2) and then multiplying by $|1+p|^{2}$ we obtain

$$
\begin{aligned}
& \left|2 z p^{\prime}+n\left(1-p^{2}\right)\right| \leq \frac{r^{2 n}|1+p|^{2}-|1-p|^{2}}{\left(1-r^{2}\right) r^{n-1}}, \\
& \left|2 z p^{\prime}\right| \leq n\left|1-p^{2}\right|+\frac{r^{2 n}|1+p|^{2}-|1-p|^{2}}{\left(1-r^{2}\right) r^{n-1}} .
\end{aligned}
$$

Thus, to prove (1) (with $\alpha=0$ ), it is sufficient to show

$$
n\left|1-p^{2}\right|+\frac{r^{2 n}|1+p|^{2}-|1-p|^{2}}{\left(1-r^{2}\right) r^{n-1}} \leq \frac{4 n r^{n} \operatorname{Re} p}{1-r^{2 n}} .
$$

Now we express $|1+p|^{2},|1-p|^{2}$ and $\operatorname{Re} p$ in terms of $\left|1-p^{2}\right|$. From $z^{n} \phi=(1-p) /(1+p)$ we obtain
(c) $|1-p|^{2}=\left|1-p^{2}\right|\left|z^{n} \phi\right|$,
(d) $|1+p|^{2}\left|z^{n} \phi\right|=\left|1-p^{2}\right| \cdot$

From (c) and (d) we have

(e) $4 \operatorname{Re} p=|1+p|^{2}-|1-p|^{2}=\left|1-p^{2}\right|\left[\left(1-\left|z^{n} \phi\right|^{2}\right) /\left|z^{n} \phi\right|\right]$.

Substituting (c), (d), (e) into (3) and then cancelling $\left|1-p^{2}\right|$ we obtain

$$
(1-|\phi|) r^{2 n-1}\left[-n\left(1-r^{2}\right)\left(1+|\phi| r^{2 n}\right)+n\left(1-r^{2 n}\right)(1+|\phi|)\right] \leq 0 .
$$

Therefore, it is sufficient to show

$$
\begin{aligned}
r\left(1-r^{2 n}\right)(1+|\phi|)- & n\left(1-r^{2}\right)\left(1+|\phi| r^{2 n}\right) \leq 0 \\
& (0 \leq r<1 ;|\phi| \leq 1 ; n=1,2,3, \ldots) .
\end{aligned}
$$

The inequality (4) is equivalent to

$$
\frac{r\left(1-r^{2 n}\right)}{n\left(1-r^{2}\right)} \leq \frac{1+|\phi| r^{2 n}}{1+|\phi|}=1-\left(1-r^{2 n}\right) \frac{|\phi|}{1+|\phi|} .
$$


For fixed $r$, the right side of (5) is minimum when $|\phi|=1$. Hence, using $|\phi|=1$ in (4) we obtain the sufficient condition

(6) $\quad n\left(1-r^{2}\right)\left(1+r^{2 n}\right)-2 r\left(1-r^{2 n}\right) \geq 0 \quad(0 \leq r<1 ; n=1,2,3, \ldots)$.

The inequality (6) is equivalent to $\left(1-r^{2}\right) M(n, r) \geq 0$, where

$$
M(n, r)=n+n r^{2 n}-2 n\left\{1+r^{2}+r^{4}+\cdots+r^{2(n-1)}\right\} .
$$

We now show that $M(n, r)>0$ for $0 \leq r<1$ and $n \geq 1$. We have $M(1, r)=$ $(1-r)^{2}>0$. For $n=2,4,6, \ldots$ we find that

$$
\begin{aligned}
M(n, r)= & 2\left[(1-r)\left(1-r^{2 n-1}\right)+\left(1-r^{3}\right)\left(1-r^{2 n-3}\right)\right. \\
& \left.+\left(1-r^{5}\right)\left(1-r^{2 n-5}\right)+\cdots+\left(1-r^{n-1}\right)\left(1-r^{n+1}\right)\right] \\
= & 2 \sum_{k=1}^{n / 2}\left(1-r^{2 k-1}\right)\left(1-r^{2 n-2 k+1}\right)>0 .
\end{aligned}
$$

For $n=3,5,7, \ldots$ we find that

$$
\begin{aligned}
M(n, r)=\left(1-r^{n}\right)^{2}+2\left[(1-r)\left(1-r^{2 n-1}\right)+\left(1-r^{3}\right)\left(1-r^{2 n-3}\right)\right. \\
\left.+\left(1-r^{5}\right)\left(1-r^{2 n-5}\right)+\cdots+\left(1-r^{n-2}\right)\left(1-r^{n+2}\right)\right] \\
=\left(1-r^{n}\right)^{2}+2 \sum_{k=1}^{(n-1) / 2}\left(1-r^{2 k-1}\right)\left(1-r^{2 n-2 k+1}\right)>0 .
\end{aligned}
$$

This completes the proof. The statement that equality is attained in (1) at $z=r$ for the given function is easily verified. We now apply Theorem 1 to obtain an extension of a result of $\mathrm{R}$. J. Libera.

Theorem 2. Let $p(z) \in P_{a, n}$. Then for $|z|=r, 0 \leq r<1, n=1,2,3, \ldots$ and any complex number $\mu, \operatorname{Re} \mu=\beta \geq 0$,

$$
\left|\frac{z p^{\prime}(z)}{p(z)-\alpha+(1-\alpha) \mu}\right| \leq \frac{2 n r^{n}}{\left(1-r^{n}\right)\left[1+\beta+(1-\beta) r^{n}\right]} .
$$

Proof. The special case of (7) with $\alpha=0$ and $n=1$ was proven by R. J. Libera [3]; we employ the same technique. Assume that $\alpha=0$, for then the transformation $p(z) \rightarrow(p(z)-\alpha) /(1-\alpha)$ will give the general result 


$$
\begin{aligned}
\left|\frac{z p^{\prime}(z)}{p(z)+\mu}\right| & \leq \frac{\left|z p^{\prime}(z)\right|}{\operatorname{Re} p(z)+\beta} \leq \frac{2 n r^{n} \operatorname{Re} p(z)}{1-r^{2 n}} \cdot \frac{1}{\operatorname{Re} p(z)+\beta} \\
& \leq \frac{2 n r^{n}}{1-r^{2 n}} \cdot \frac{1}{1+\beta /|p(z)|},
\end{aligned}
$$

where we have used the inequality (1) (with $\alpha=0$ ). The result now follows by substituting $|p(z)|=\left|\left(1-z^{n} \phi\right) /\left(1+z^{n} \phi\right)\right| \leq\left(1+r^{n}\right) /\left(1-r^{n}\right)$.

In Theorem 2 , the choice $\mu=(\alpha-c) /(1-\alpha)$ yields

$$
\begin{aligned}
\left|\frac{z p^{\prime}(z)}{p(z)-c}\right| \leq \frac{2 n(1-\alpha) r^{n}}{\left(1-r^{n}\right)\left[1-\gamma+(1-2 \alpha+\gamma) r^{n}\right]} \\
\left(p(z) \in P_{a, n} ; 0 \leq r<1 ; \gamma=\operatorname{Re} c \leq \alpha\right) .
\end{aligned}
$$

If we integrate the inequality (7) along the line segment joining the origin and the point $z$, we obtain

Corollary 1. With the same hypotheses as in Theorem 2, we have

$$
\begin{aligned}
\left|\log \frac{p(z)-\alpha+(1-\alpha) \mu}{(1-\alpha)(1+\mu)}\right| & \leq \log \frac{(1+\beta)+(1-\beta) r^{n}}{(1+\beta)\left(1-r^{n}\right)}, \\
\left|\frac{p(z)-\alpha+(1-\alpha) \mu}{(1-\alpha)(1+\mu)}\right| & \leq \frac{(1+\beta)+(1-\beta) r^{n}}{(1+\beta)\left(1-r^{n}\right)}
\end{aligned}
$$

In (7b), the choice $\mu=(\alpha-c) /(1-\alpha)$ yields

$$
|p(z)-c| \leq \frac{(1-\operatorname{Re} c)+(1-2 \alpha+\operatorname{Re} c) r^{n}}{(1-\operatorname{Re} c)\left(1-r^{n}\right)} \quad(\operatorname{Re} c \leq \alpha) .
$$

Finally, we note that in Theorem 2 the choice $\mu=\alpha /(1-\alpha)$ yields the theorem which was recently announced by Dorothy B. Shaffer [6].

We now apply Theorem 2 to obtain a sharp result involving the partial sums of functions belonging to the class $(K)$.

Theorem 3. Let $f(z)=z+a_{2} z^{2}+\cdots \epsilon(K)$, and $S_{n}(z)=z+a_{2} z^{2}+\cdots+$ $a_{n} z^{n}$ its partial sums. Then for $n=1,2,3, \ldots$ and $|z|=r<1$,

$$
\left|z f^{\prime}(z) / f(z)-z S_{n}^{\prime}(z) / S_{n}(z)\right| \leq n r^{n} /\left(1-r^{n}\right) .
$$

Equality is attained, for each $n$, at $z=r$ for the function $f(z)=z /(1-z)$. 
Proof. It has been shown independently by this writer [2] and by $\mathrm{T}$. Sheil-Small [7] that if $f(z) \in(K)$ and $S_{n}(z)$ are its partial sums, then $\mid f(z)-$ $S_{n}(z)|\leq| z^{n} f(z) \mid$ and this implies $\operatorname{Re}\left[f(z) / S_{n}(z)\right]>1 / 2$ for $n=1,2,3, \cdots$ and $|z|<1$. Thus, since

$$
p(z)=f(z) / S_{n}(z)=1+a_{n+1} z^{n}+\cdots \in P_{1 / 2, n},
$$

the result ( 8 ) follows readily upon substituting (9) into the inequality (7) of Theorem 2, taking $\mu=1$ and $\alpha=1 / 2$. For the function $f(z)=z /(1-z)$ we have

$$
z f^{\prime}(z) / f(z)-z S_{n}^{\prime}(z) / S_{n}(z)=n z^{n} /\left(1-z^{n}\right),
$$

so that for this function equality is attained in (8) at $z=r$.

If in (7a) we take $\mu=1, \alpha=1 / 2, p(z)=f(z) / s_{n}(z)$ we obtain

Corollary 2. Let $f(z) \in(K)$, and $S_{n}(z)$ its partial sums. Then for $n=$ $1,2,3, \ldots$ and $|z|=r<1$,

$$
\left|\log \left(f(z) / S_{n}(z)\right)\right| \leq \log \left(1 /\left(1-r^{n}\right)\right)
$$

We now apply Theorem 3 to obtain the radius of univalence and starlikeness for the partial sums of a convex function.

Theorem 4. Let $f(z)=z+a_{2} z^{2}+\cdots \in(K)$, and $S_{n}(z)=z+a_{2} z^{2}+\cdots+$ $a_{n} z^{n}$ its partial sums. Then $S_{n}(z)$ is univalent and starlike in the disk $|z|$ $<r_{0}$, where $r_{0}$ is the positive root of the equation

$$
1-(1+n) r^{n}-n r^{n+1}=0 \quad(n \geq 2) .
$$

This result is sharp for each even $n=2,4,6, \cdots$ for the function $f(z)=$ $z /(1-z)$.

Proof. From (8) of Theorem 3 we have

$$
\operatorname{Re}\left(z S_{n}^{\prime}(z) / S_{n}(z)\right) \geq \operatorname{Re}\left(z f^{\prime}(z) / f(z)\right)-n r^{n} /\left(1-r^{n}\right) .
$$

Since $f \in(K)$, we apply the well-known bound $\operatorname{Re}\left(z f^{\prime} / f\right) \geq 1 /(1+r)$, so that

$$
\operatorname{Re} \frac{z S_{n}^{\prime}(z)}{S_{n}(z)} \geq \frac{1}{1+r}-\frac{n r^{n}}{1-r^{n}}=\frac{1-(1+n) r^{n}-n r^{n+1}}{(1+r)\left(1-r^{n}\right)} \geq 0
$$

if $1-(1+n) r^{n}-n r^{n+1} \geq 0$. For the function $f(z)=z /(1-z) \in(K)$, we have

$$
z S_{n}^{\prime}(z) / S_{n}(z)=\left(1-(1+n) z^{n}+n z^{n+1}\right) /(1-z)\left(1-z^{n}\right) .
$$


For $z=-r$ and $n=2,4,6, \ldots$ the right side of (12) is identical with the right side of (11) so that for $n$ even no improvement of (10) is possible in the class $(K)$. We note that for each $n, r_{0}$ given by $(10)$ satisfies $r_{0} \geq 1 / 2$ and, furthermore, $r_{0} \rightarrow 1$ as $n \rightarrow \infty$.

\section{REFERENCES}

1. S. D. Bernardi, The radius of univalence of certain analytic functions, Proc. Amer. Math. Soc. 24 (1970), 312-318. MR 40 \#433.

2. - Univalent convex maps of the unit disk. I, II, III, Notices Amer. Math. Soc. 17 (1970), 441, 566; ibid. 18 (1971), 640. Abstract \#70T-B54; 70T-B77; $71 \mathrm{~T}-\mathrm{B} 113$.

3. R. J. Libera, Some radius of convexity problems, Duke Math. J. 31 (1964), 143-158. MR 28 \#4099.

4. T. H. MacGregor, The radius of univalence of certain analytic functions, Proc. Amer. Math. Soc. 14 (1963), 514-520. MR 26 \#6388.

5. Z. Nehari, Conformal mapping, McGraw-Hill, New York, 1952. MR 13, 640.

6. D. B. Shaffer, Distortion theorems for a special class of analytic functions, Notices Amer. Math. Soc. 19 (1972), A519. Abstract \#72T-B152.

7. T. Sheil-Small, A note on the partial sums of convex schlicht functions, Bull. London Math. Soc. 2 (1970), 165-168. MR 42 \#485.

COURANT INSTITUTE OF MATHEMATICAL SCIENCES, NEW YORK UNIVERSITY, NEW YORK, NEW YORK 10012 